\title{
Transfusion in children with acute respiratory distress syndrome
}

\author{
Tine François ${ }^{1}$, Guillaume Emeriaud ${ }^{1}$, Oliver Karam $^{2}$, Marisa Tucci ${ }^{1}$ \\ ${ }^{1}$ Department of Pediatrics, Division of Pediatric Critical Care Medicine, Centre Hospitalier Universitaire Sainte-Justine, Université de Montréal, \\ Montréal, Québec, Canada; ${ }^{2}$ Division of Pediatric Critical Care Medicine, Children's Hospital of Richmond at VCU, Richmond, VA, USA \\ Contributions: (I) Conception and design: T François, G Emeriaud, M Tucci; (II) Administrative support: T François, G Emeriaud, M Tucci; (III) \\ Provision of study materials or patients: All authors; (IV) Collection and assembly of data: All authors; (V) Data analysis and interpretation: All \\ authors; (VI) Manuscript writing: All authors; (VII) Final approval of manuscript: All authors. \\ Correspondence to: Marisa Tucci, MD. Centre Hospitalier Universitaire (CHU) Sainte-Justine, 3175, Chemin de la Côte Sainte-Catherine, Montréal, \\ Québec, H3T 1C5, Canada. Email: marisa.tucci@recherche-ste-justine.qc.ca.
}

\begin{abstract}
Transfusion is a frequent treatment in pediatric patients with acute respiratory distress syndrome (PARDS) although evidence to support transfusion decision-making is lacking. The purpose of this review is to review the current state of knowledge on the issue of transfusion in children with PARDS and to detail the possible beneficial effects and potential deleterious impacts of transfusion in this patient population. Based on the current literature and recent guidelines, a restrictive red blood cell (RBC) transfusion strategy (avoidance of transfusion when the haemoglobin level is above $7 \mathrm{~g} / \mathrm{dL}$ ) is indicated in stable patients without severe PARDS, as these were excluded from the large trials. In children with severe PARDS, further research is needed to determine if factors other than the haemoglobin level might guide RBC transfusion decisionmaking by better characterizing the presence of low oxygen delivery $\left(\mathrm{DO}_{2}\right)$. Additionally, appropriate indications for prophylactic transfusion of hemostatic products (plasma or platelets) in children with PARDS are lacking.
\end{abstract}

Keywords: Acute respiratory distress syndrome (ARDS); blood transfusion; child; pediatric intensive care; blood transfusion

Submitted Jul 08, 2019. Accepted for publication Aug 01, 2019.

doi: 10.21037/atm.2019.08.28

View this article at: http://dx.doi.org/10.21037/atm.2019.08.28

\section{Introduction}

Pediatric acute respiratory distress syndrome (PARDS) is a heterogeneous syndrome that involves severe acute inflammatory lung injury in children and can be triggered by pulmonary and/or extrapulmonary factors. Although it represents a relatively small percentage of the total number of pediatric intensive care unit (PICU) admissions, morbidity and mortality associated with ARDS are significant and clinical management is challenging (1). ARDS progresses from inflammatory to proliferative and then fibrotic phases and is characterized by rapidly progressive hypoxemic respiratory failure caused by widespread inflammation with injury to the pulmonary epithelium and endothelium that leads to loss of the permeability barrier and subsequent alveolar edema $(1,2)$.
This process is accompanied by surfactant dysfunction and alterations in the hemostatic balance that favors a procoagulant state possibly leading to micro-thrombi and pulmonary hypertension (1).

This clinical condition poses significant threat to patients of all age groups including children, but several adult ARDS definitions, reported over the last 5 decades, were not applicable to children (3). In 2015, the Pediatric Acute Lung Injury Consensus Conference (PALICC) published a pediatricspecific definition for ARDS in children, and proposed specific patient management recommendations for PARDS as well as priorities for potential future research (1). PALICC included transfusion among the possible therapeutic approaches, but emphasized the lack of definitive data to guide the use of these therapies in patients with PARDS $(1,4)$. The international Pediatric Acute Respiratory Distress Incidence 
and Epidemiology (PARDIE) study, an observational, crosssectional study of new cases of PARDS (based on PALICC definition), reported an overall prevalence of $3.2 \%$ in a total of 23,280 PICU admissions. Mortality associated with PARDS varies from $17 \%$ to $27 \%$, depending on the definition used to identify PARDS $(1,5,6)$.

Anemia is a common complication in critically ill patients and more likely to occur in hypoxemic patients with ARDS who may require red blood cell (RBC) transfusion as part of supportive care to improve oxygen transport. Anemia results from two possible processes: (I) excessive blood loss due to frequent blood sampling, hemorrhage, or a shortened RBC circulatory life span and (II) diminished RBC production believed to be secondary to inflammatory processes leading to impaired RBC proliferation, iron metabolism, erythropoietin production, and signaling (7). In addition, patients with ARDS might also develop thrombocytopenia, coagulation disorders and other complications that might require administration of other blood components, including platelets, plasma and other components (cryoprecipitate, coagulation factors).

Multiple blood products, both cellular (i.e., RBCs and platelets) and acellular (fresh-frozen plasma and cryoprecipitate) products, are transfused in children. RBC products make up approximately two thirds of transfused products with the remainder comprised of plasma, platelets, cryoprecipitate, and granulocytes (8). Data describing current utilization of blood products and temporal trends describing the incidence of transfusion in children are scarce. Some data have reported large decreases in pediatric transfusions (8) while others report a mild increase in RBC transfusions in patients less than 18 years of age (9). Data describing utilization of blood products in children with PARDS is limited at the present time. It is known that transfusions are frequent in PICU patients. Data from before 2010 indicates that at least one RBC transfusion is administered in $17 \%$ to $50 \%$ of PICU admissions $(10,11)$; more recent data on RBC transfusion in PICU is currently not available. In addition, the incidence of transfusions of other blood products is less well known (12). One observational single center study reported an incidence of $7.1 \%$ for platelet transfusion in PICU patients (13). Point prevalence studies have been carried out to estimate the prevalence of plasma and platelet transfusion in critically ill children $(14,15)$. More than two-thirds of pediatric critical care physicians prescribe plasma transfusions for nonbleeding critically ill children with moderately abnormal coagulopathy and only $22 \%$ of critically ill children receive plasma for critical bleeding (14).

Transfusion of blood products in critically ill children with PARDS is standard of care, especially in those with severe disease and numerous comorbidities (16). In the recent years, there has been increasing awareness concerning transfusion indications and complications in critically ill children $(14,15,17,18)$. It is also becoming increasingly evident that pediatric patients have unique physiologic characteristics that may affect their reactions to blood products resulting in higher rates of transfusion reactions compared to adults (19). Inappropriate blood transfusions can cause harm and sometimes worsen patient outcomes and among the most frequent adverse effects of transfusion is the worsening of respiratory function.

An important clinical question therefore frequently arises for the bedside clinicians: should a patient with PARDS receive RBCs to improve oxygen delivery $\left(\mathrm{DO}_{2}\right)$ or be administered other blood products to correct coagulation abnormalities, or do the side effects of transfusion outweigh the potential benefits?

In this comprehensive review we will list the potential beneficial effects as well as the possible deleterious impact of transfusion of blood products in critically ill children with a focus on the respiratory dysfunction and review the existing evidence for an association between transfusion and PARDS outcome. This will lead us to formulate some clinical suggestions on transfusion strategy in patients with PARDS.

\section{Effect of transfusion on the pulmonary condition of critically ill children}

Potential positive and negative effects of transfusion on the respiratory function in critically ill children are listed in Table 1.

\section{Potential beneficial effects of transfusion}

The most frequent indications to transfuse RBCs are restoration of blood volume in hemorrhagic shock and correction of isovolemic anemia. In critically children, the most important reason evoked to transfuse RBCs is the expectation that $\mathrm{DO}_{2}$ will improve and thereby prevent development of shock (anaerobic metabolism) by increasing oxygen consumption $\left(\mathrm{VO}_{2}\right)(20)$. Most studies that have measured the effect of improved $\mathrm{DO}_{2}$ have been conducted in patients with sepsis and have failed to demonstrate a beneficial effect of RBC transfusion on measured systemic 
Table 1 Schematic listing of reasons in favor or against use of transfusion in children with respiratory dysfunction

\begin{tabular}{|c|c|}
\hline Favoring transfusion & Against transfusion \\
\hline Increased oxygen affinity (due to 2,3-DPG depletion) & Transfusion-related circulatory overload (TACO) \\
\hline Improvement of oxygen delivery $\left(\mathrm{DO}_{2}\right)$ & Respiratory dysfunction associated with transfusion \\
\hline Improved hemodynamic status & Risk of ventilator-acquired pneumonia and ventilator-acquired event \\
\hline Improvement of pulmonary hypertension & Anaphylactic and hemolytic transfusion reactions \\
\hline \multirow{4}{*}{$\begin{array}{l}\text { Improvement of coagulopathy and reduced risk of bleeding } \\
\text { (e.g., pulmonary hemorrhage) }\end{array}$} & Bacterial contamination and transfusion-related sepsis \\
\hline & $\begin{array}{l}\text { Transfusion-associated graft-versus-host disease and transfusion-associated } \\
\text { microchimerism }\end{array}$ \\
\hline & Risk of prolonged length of stay in ICU \\
\hline & Risk of prolonged ventilation \\
\hline
\end{tabular}

$\mathrm{RBC}$, red blood cell.

oxygen uptake (21-23). In a small observational study of adult ARDS patients there was no change in $\mathrm{VO}_{2}$ after transfusion, but an increase in $\mathrm{DO}_{2}$ without changes in cardiac output, as well as an increased mixed venous oxygen saturation were noted (24). Two reviews have questioned the possible positive effect of RBC transfusion on tissue perfusion and tissue oxygenation and could not conclusively report any benefit with regard to $\mathrm{O}_{2}$ consumption $(23,25)$.

Hemodynamic instability might also be a trigger to transfuse critically ill patients, or to improve volemic status in patients with high ventilation pressures. Transfusion for these indications seems to be largely abandoned.

The possible benefit of RBC transfusion on pulmonary hypertension is also more a matter of clinical judgement than a scientifically proven indication, as transfusion can in fact worsen pulmonary hypertension (16).

Common indications for hemostasis-related transfusion in patients with PARDS include treatment of thrombocytopenia or coagulopathy, active bleeding as well as prevention of bleeding $(15,18)$. Although it seems appropriate to try to prevent pulmonary hemorrhage in patients with PARDS, there is no evidence to support prophylactic administration of plasma. Similarly, the majority of platelet transfusions in critically ill children are given prophylactically to nonbleeding patients, with significant variation in platelet thresholds (15). Appropriate indications for prophylactic platelet transfusion are currently unclear and it is essential to determine when the benefits outweigh the side effects.

\section{Potential deleterious impacts}

A few studies have been published suggesting that RBC transfusion in critically ill children with or without acute lung injury (ALI) or ARDS is associated with increased morbidity and mortality $(11,26)$. Transfusion can potentially increase pulmonary morbidity by several mechanisms leading to inflammatory injury and causing hypoxemia and respiratory distress. This reaction can lead to a condition referred to as transfusion-related ALI or TRALI. Classic TRALI is a syndrome defined by the acute onset ( $\leq 6$ hours following transfusion) of new hypoxemia (with a $\mathrm{PaO}_{2} /$ $\mathrm{FiO}_{2} \leq 300$ or $\mathrm{SpO}_{2}<90 \%$ on room air), bilateral infiltrates on chest X-ray, no evidence of left atrial hypertension, no pre-existing ALI and should have no temporal relationship with alternative ALI risk factors, if any are present (27). The epidemiology of TRALI in children is not well known, because it is frequently underreported (28); consequently, the reported incidence of TRALI in children varies 
from $2 / 100,000$ transfusions (28) to $6 / 100,000(29,30)$ to $89 / 100,000$ (31). A higher prevalence of TRALI has been reported after transfusion of platelets or plasma (31). Several pathophysiological mechanisms can be involved in the occurrence of TRALI. Most cases of definite TRALI in non-critically ill patients were attributable to donor antibodies that react with leukocytes of the receiver (32). This mechanism seems less frequent since selection of blood donors has been optimized. A second pathophysiological mechanism of TRALI was first suggested in an animal model by Silliman et al. to be the result of two hits, similar to the pathophysiology of ARDS (33). The first hit is related to the clinical condition of the transfusion recipient, which is frequently associated with a systemic inflammatory status during critical illness (34). The second hit on the pulmonary tissue is caused by pro-inflammatory or bioactive factors in the plasma fraction of stored blood, such as lipids, cytokines, leukoagglutinins, or other intrinsic factors (IL-6, IL-8, leukocyte antibodies, lysophosphatidylcholines). Both can result in lung damage because of an interaction of the pulmonary endothelium with activated circulating neutrophils $(31,33,35)$. A retrospective study observed a $0.5 \%$ incidence of 2,660 ARDS cases in 488,381 pediatric patients following trauma (36). Transfusion was associated with the occurrence of ARDS after trauma but was not an independent risk factor. Mulder et al. retrospectively analysed in 2,015 transfused critically ill children and a possible association with ALI and observed an incidence of TRALI of $6.9 \%$ (30). Patients with TRALI had significantly higher mortality $(76 \%$ vs. $11 \%, \mathrm{P}<0.001)$ and a longer median duration of ventilation [183 (interquartile range, 52-282) vs. 25 (0-139) hours, $\mathrm{P}<0.002$ ], after controlling for severity of illness; the important variations in this data suggest the presence of a reporting or detection bias. Importantly, 90\% of the studied patients who developed TRALI also had another risk factor for ALI (i.e., possible TRALI which is defined as ALI occurring in the presence of an alternative risk factor) and there was no subgroup analysis of the patients with pre-existing ARDS. Mechanical ventilation at the time of transfusion was an independent risk factor for developing TRALI (30).

Use of currently available TRALI definitions in critically ill children is questionable (37) because thresholds for various criteria are not applicable and because pre-existing ALI is an exclusion criterion. Respiratory dysfunction, which is present in more than half the patients that receive a transfusion in PICU, should not preclude them from being diagnosed with TRALI (38). For this reason, Kleiber et al. introduced the concept of respiratory dysfunction associated with RBC transfusion (RDAT) to describe deterioration of respiratory function after transfusion regardless of baseline respiratory status (38). Moreover, acute lung inflammatory injury occurring post transfusion comprises a broad spectrum that includes classical TRALI, possible TRALI (see above), and delayed TRALI (occurring between 6-72 hours after transfusion) (39).

The volume of blood products transfused can also have an adverse effect on the pulmonary condition of critically ill patients. Transfusion-associated circulatory overload (TACO) is a more frequent transfusion complication than TRALI, according to hemovigilance data. De Cloedt et al. reported an overall incidence of TACO varying from $1.5 \%$ to $76 \%$ in PICU, depending on the definition used (40). TACO is differentiated from TRALI as it is characterised by signs of fluid overload including tachycardia, hypertension, pulmonary edema, positive fluid balance and elevated levels of brain natriuretic peptides (BNP). In critically ill children, clinical differentiation between TACO and the TRALIspectrum is challenging because overlap in the definitions and pre-existing risk factors are frequently present for both conditions (41).

Bacterial contamination of stored blood products resulting in infection as well as the risk of developing ventilator-associated pneumonia (VAP) or ventilatorassociated events (VAE) are other potential transfusionrelated complications. A recent review article by Muszynski et al. summarizes current knowledge on transfusionrelated immunomodulation (TRIM) (42). The review outlines the immunologic response that occurs in critical illness and provides clinical evidence in support of immunomodulatory effects of blood product transfusion. Among the mechanisms that could explain post-transfusion immunoparalysis is a possible two-hit model, involving pre-disposing immunologic issues present prior to transfusion and additional changes occurring following transfusion (41-45).

Additional risks of transfusion include the possible development of transfusion-associated graft-versus-host disease and transfusion-associated microchimerism resulting from the transfusion of immunocompetent cells (or DNA) into an immunoincompetent patient (43).

Among other potential transfusion-related complications are changes in rheology and altered vasoactivity secondary to dysregulation of nitric oxide (NO). Reynolds et al. has reported a suppressive effect of transfused RBCs on S-nitrosothiols (SNO) which could augment ventilation- 
perfusion mismatch and potentially increase pulmonary vascular resistance due to unopposed vasoconstriction (44). This too could lead to a worsening oxygenation and a need for increased ventilatory support leading to increased risk of ventilator-induced lung injury. Riccio et al. found that $\mathrm{RBC}$ renitrosylation by using $\mathrm{NO}$ donors is promising in correcting the deficient properties (e.g., adhesivity, rigidity and SNO loss) of banked RBCs and in turn improving outcomes after transfusion (45). More recently some authors have reported that circulating $\mathrm{RBC}$-derived microparticles are associated with altered plasma vasoactivity and abnormal vasoregulation after RBC or plasma transfusion $(46,47)$.

Thus, while transfusions are frequent in PICU, there are little data that demonstrate a beneficial impact of $\mathrm{RBC}$ transfusion on $\mathrm{VO}_{2}$ or on respiratory function in critically ill children, except in those with very severe anemia [i.e., a hemoglobin $(\mathrm{Hb})$ level $<5 \mathrm{~g} / \mathrm{dL}$ ] in whom the risk of mortality increases significantly if not transfused (48-50). Moreover, there is also little data indicating whether plasma and platelet transfusion for nonbleeding patients in PICU causes benefit or harm. Contrastingly, accumulating evidence suggests that transfusions are associated with a deterioration of respiratory function. Recognition of these transfusion-related adverse events remains challenging.

\section{Existing evidence regarding transfusion and outcome in children with PARDS}

The epidemiology of transfusion in PARDS is not well known and has been studied in a few retrospective studies (16,51-53) and one large prospective point prevalence study (6) . Most studies evaluating transfusion in the intensive care unit have not included patients with preexisting ALI/ARDS or were conducted only in adult patients. In a retrospective cohort study of pediatric patients with ALI/ARDS, Rajasekaran et al. retrospectively studied the course of 34 transfused patients and compared them to 45 non-transfused control patients admitted to the PICU (51). They observed that early transfusions within the first 48 hours in patients with ALI/ARDS were associated with increased ventilatory needs. Patients who needed high frequency oscillatory ventilation or extracorporeal membrane oxygenation (ECMO) in the first $48 \mathrm{~h}$ of mechanical ventilation, who had pulmonary hypertension or pre-existing multiple organ dysfunction syndrome (MODS) at intubation were excluded from this retrospective case-control study. A longer mean duration of ventilation and delayed recovery in transfused patients were observed $(15.6 \pm 1.7$ vs. $9.5 \pm 0.6$ ventilator days in control, with $\mathrm{P}<0.001)(51)$.

In a retrospective analysis of an epidemiologic cohort study, Church et al. assessed the effect of the different blood products when transfused in 315 pediatric patients with ALI (52). Transfusion of FFP was associated with an increased mortality and increased ventilator days, although no effect of RBC or platelet transfusion on outcome were noted. A more positive fluid balance over the first 72 hours was observed in transfused ALI-patients $(\mathrm{P}=0.03)$, which may lead to a potential overlap with the diagnosis of TACO. Because of the observational design, it is possible that blood transfusions were in fact a surrogate for the patients' underlying severity of illness.

Zubrow et al. undertook a retrospective analysis of 357 patients who had been prospectively enrolled in a cohort study aiming to characterize degree of lung injury in pediatric ARDS. Among those transfused, severity of illness prior to transfusion was higher; $43 \%$ had received RBCs and $25 \%$ had received FFP or platelets (16). Transfused PARDS patients also had lower $\mathrm{PaO}_{2} / \mathrm{FiO}_{2}$ ratios at 24 hours after PARDS diagnosis $(\mathrm{P}<0.05)$, were more often immunocompromised $(\mathrm{P}<0.001)$ and had more nonpulmonary organ dysfunction $(\mathrm{P}<0.001)$. Overall these transfused patients had a worse survival and longer duration of ventilation by univariate analysis. After controlling for confounders, these authors reported that neither RBC, nor FFP or platelet transfusion were shown to be associated with higher mortality. However, a great volume of RBC transfusions was shown to be associated with increased mortality and increased ventilator days among survivors.

Similar observations were made by Emeriaud et al. in 2018 in their prospective PARDS cohort of 27 patients, with almost half of the moderate and severe PARDS receiving at least one $\mathrm{RBC}$ transfusion during admission (respectively 3 in 6 patients and 7 out of 15 patients). Transfused patients also had a higher PRISM III score and higher oxygen saturation index (53).

In summary, all these observational studies confirm an association between transfusion and adverse outcome in children with PARDS but do not allow for demonstration of a causal relationship due to their design. In all these studies, transfusion was consistently associated with a greater severity of illness at baseline. At least three hypotheses could therefore explain such an association: (I) the sickest patients are more likely to require transfusion; (II) the sickest patients are also more likely to develop worsening 
respiratory dysfunction; or (III) physicians more frequently decide to transfuse patients with severe or deteriorating ALI.

\section{When should we transfuse a patient with PARDS?}

Because of the lack of specific studies in pediatric patients with ARDS/ALI, it is difficult to generate clear recommendations about transfusion in this specific group of patients. Additional studies are required to best inform clinicians with regard to the risk-to-benefit ratio of transfusion in these patients. Blood transfusions should only be administered after careful consideration of the patient's unique risk for transfusion complications and the anticipated physiologic benefits of the planned transfusion.

With regard to RBC transfusion, it would seem that a restrictive strategy could be acceptable for most critically ill patients, although best practice is undetermined for patients with severe ARDS. Clinical recommendations for RBC transfusions in critically ill children with respiratory failure were recently made by the Pediatric Critical Care Transfusion and Anemia Expertise Initiative. They strongly recommend administering an $\mathrm{RBC}$ transfusion when the $\mathrm{Hb}$ level drops below $5 \mathrm{~g} / \mathrm{dL}$, but to not systematically transfuse hemodynamically stable children with respiratory failure and a $\mathrm{Hb}$ level $\geq 7 \mathrm{~g} / \mathrm{dL}(1,54,55)$. No strong recommendation was made for patients with severe hypoxemia (54).

In patients for whom no strong recommendation exists (severe PARDS or hemodynamically unstable patients), it would seem best not to base a decision to transfuse solely on $\mathrm{Hb}$ level but rather to factor in other clinical and physiological parameters that might indicate low $\mathrm{DO}_{2}$, e.g., elevation of lactate level, low central venous oxygenation, or low near infrared spectroscopy (NIRS) level (56). If there is no evidence of low $\mathrm{DO}_{2}$, it is likely that a restrictive transfusion strategy is safe. Such management would minimize transfusion-related side effects and therefore optimize the balance between possible benefits versus potential deleterious effects of transfusion on the pulmonary condition of the patient.

For other blood products, it remains difficult to provide clear recommendations as evidence is lacking. Appropriate indications for prophylactic platelet transfusion (if platelets $<10,000 / \mu \mathrm{L}$ ), plasma transfusion (INR $>2.5$ ) or cryoprecipitate infusion (if fibrinogen $<75-100 \mathrm{mg} / \mathrm{dL}$ ) remain unclear. Similar to RBC transfusion, other factors (e.g., volume status, risk of bleeding) have to be accounted for to ascertain whether the benefits outweigh the side effects (43).

Thus, if a decision is made to transfuse patients with respiratory dysfunction, it is particularly important to transfuse smaller volumes per dose without increasing patient exposure to blood products, to institute therapy to limit or prevent fluid overload (other fluid restriction and diuretics) (57) and to carefully monitor for potential respiratory complications such as TRALI, TACO, or RDAT.

\section{Conclusions}

Careful blood management is imperative in patients with PARDS. The presence of inflammatory lung injury in children with a severe prognosis and a clinical context which often mandates blood transfusion warrant careful consideration. Blood products are frequently considered and administered in this patient population. Potential benefits of transfusion, and in particular the impact on $\mathrm{DO}_{2}$ following transfusion in PARDS patients remain to be demonstrated. On the other hand, accumulating evidence suggests possible transfusion-associated pulmonary, circulatory and inflammatory deleterious effects. Restrictive $\mathrm{RBC}$ transfusion strategy seems to be the best option in the majority of critically ill patients. Appropriate indications for prophylactic transfusion of other blood products are unclear and require clinical judgment. Further research is needed to guide optimal transfusion practices in children with severe ARDS. More robust hemovigilance data, more appropriate definitions and careful analysis of data in critically ill children are required to improve the understanding of the causes, occurrence and clinical outcomes associated with transfusion in critically ill children with PARDS.

\section{Acknowledgments}

None.

\section{Footnote}

Conflicts of Interest: The authors have no conflicts of interest to declare.

Ethical Statement: The authors are accountable for all aspects of the work in ensuring that questions related to the accuracy or integrity of any part of the work are appropriately investigated and resolved. 


\section{References}

1. Pediatric Acute Lung Injury Consensus Conference Group. Pediatric acute respiratory distress syndrome: consensus recommendations from the Pediatric Acute Lung Injury Consensus Conference. Pediatr Crit Care Med 2015;16:428-39.

2. Ware LB, Matthay MA. The acute respiratory distress syndrome. N Engl J Med 2000;342:1334-49.

3. Cheifetz IM. Pediatric ARDS. Respir Care 2017;62:718-31.

4. Valentine SL, Nadkarni VM, Curley MA, et al. Nonpulmonary treatments for pediatric acute respiratory distress syndrome: proceedings from the Pediatric Acute Lung Injury Consensus Conference. Pediatr Crit Care Med 2015;16:S73-85.

5. ARDS Definition Task Force, Ranieri VM, Rubenfeld GD, et al. Acute respiratory distress syndrome: the Berlin Definition. JAMA 2012;307:2526-33.

6. Khemani RG, Smith L, Lopez-Fernandez YM, et al. Paediatric acute respiratory distress syndrome incidence and epidemiology (PARDIE): an international, observational study. Lancet Respir Med 2019;7:115-28.

7. Hayden SJ, Albert TJ, Watkins TR, et al. Anemia in critical illness: insights into etiology, consequences, and management. Am J Respir Crit Care Med 2012;185:1049-57.

8. Jones J. National Blood Collection and Utilization in the United States, 2017 [Internet]. Office of Blood, Organ, and Other Tissue Safety Division of Healthcare Quality Promotion National Center for Emerging and Zoonotic Infectious Diseases Centers for Disease Control and Prevention; 2018. Available online: https:// aabb.confex.com/aabb/2018/mediafile/Handout/ Session2403/MN2-14.pdf

9. Goel R, Chappidi MR, Patel EU, et al. Trends in Red Blood Cell, Plasma, and Platelet Transfusions in the United States, 1993-2014. JAMA 2018;319:825-7.

10. Demaret P, Tucci M, Ducruet T, et al. Red blood cell transfusion in critically ill children (CME). Transfusion 2014;54:365-75; quiz 364.

11. Bateman ST, Lacroix J, Boven K, et al. Anemia, blood loss, and blood transfusions in North American children in the intensive care unit. Am J Respir Crit Care Med 2008;178:26-33.

12. Du Pont-Thibodeau G, Tucci M, Ducruet T, et al. Survey on stated transfusion practices in PICUs. Pediatr Crit Care Med 2014;15:409-16.

13. Du Pont-Thibodeau G, Tucci M, Robitaille N, et al.
Platelet Transfusions in Pediatric Intensive Care. Pediatr Crit Care Med 2016;17:e420-9.

14. Karam O, Tucci M, Lacroix J, et al. International survey on plasma transfusion practices in critically ill children. Transfusion 2014;54:1125-32.

15. Nellis ME, Karam O, Mauer E, et al. Platelet Transfusion Practices in Critically Ill Children. Crit Care Med 2018;46:1309-17.

16. Zubrow ME, Thomas NJ, Friedman DF, et al. Red blood cell transfusions are associated with prolonged mechanical ventilation in pediatric acute respiratory distress syndrome. Pediatr Crit Care Med 2018;19:e88-96.

17. Lacroix J, Hébert PC, Hutchison JS, et al. Transfusion strategies for patients in pediatric intensive care units. $\mathrm{N}$ Engl J Med 2007;356:1609-19.

18. Karam O, Demaret $P$, Shefler A, et al. Indications and Effects of Plasma Transfusions in Critically Ill Children. Am J Respir Crit Care Med 2015;191:1395-402.

19. Vossoughi S, Perez G, Whitaker BI, et al. Analysis of pediatric adverse reactions to transfusions. Transfusion 2018;58:60-9.

20. Laverdière C, Gauvin F, Hébert PC, et al. Survey on transfusion practices of pediatric intensivists. Pediatr Crit Care Med 2002;3:335-40.

21. Marik PE, Bankov A. Sublingual capnometry versus traditional markers of tissue oxygenation in critically ill patients. Crit Care Med 2003;31:818-22.

22. Napolitano LM, Kurek S, Luchette FA, et al. Clinical practice guideline: red blood cell transfusion in adult trauma and critical care. Crit Care Med 2009;37:3124-57.

23. Roberson RS, Bennett-Guerrero E. Impact of red blood cell transfusion on global and regional measures of oxygenation. Mt Sinai J Med 2012;79:66-74.

24. Ronco JJ, Phang PT, Walley KR, et al. Oxygen consumption is independent of changes in oxygen delivery in severe adult respiratory distress syndrome. Am Rev Respir Dis 1991;143:1267-73.

25. Tsai AG, Hofmann A, Cabrales P, et al. Perfusion vs. oxygen delivery in transfusion with "fresh" and "old" red blood cells: the experimental evidence. Transfus Apher Sci 2010;43:69-78.

26. Gong MN, Thompson BT, Williams P, et al. Clinical predictors of and mortality in acute respiratory distress syndrome: potential role of red cell transfusion. Crit Care Med 2005;33:1191-8.

27. Kleinman S, Caulfield T, Chan P, et al. Toward an understanding of transfusion-related acute lung injury: statement of a consensus panel. Transfusion 
2004;44:1774-89.

28. Gauvin F, Robillard P, Hume H, et al. Transfusion-related acute lung injury in the Canadian paediatric population. Paediatr Child Health 2012;17:235-9.

29. Lieberman L, Petraszko T, Yi Q, et al. Transfusion-related lung injury in children: a case series and review of the literature. Transfusion 2014;54:57-64.

30. Mulder HD, Augustijn QJ, van Woensel JB, et al. Incidence, risk factors, and outcome of transfusion-related acute lung injury in critically ill children: a retrospective study. J Crit Care 2015;30:55-9.

31. Silliman CC, Boshkov LK, Mehdizadehkashi Z, et al. Transfusion-related acute lung injury: epidemiology and a prospective analysis of etiologic factors. Blood 2003;101:454-62.

32. Kopko PM, Marshall CS, MacKenzie MR, et al. Transfusion-related acute lung injury: report of a clinical look-back investigation. JAMA 2002;287:1968-71.

33. Silliman CC, Voelkel NF, Allard JD, et al. Plasma and lipids from stored packed red blood cells cause acute lung injury in an animal model. J Clin Invest 1998;101:1458-67.

34. Proulx F, Joyal JS, Mariscalco MM, et al. The pediatric multiple organ dysfunction syndrome. Pediatr Crit Care Med 2009;10:12-22.

35. Sanchez R, Toy P. Transfusion related acute lung injury: a pediatric perspective. Pediatr Blood Cancer 2005;45:248-55.

36. de Roulet A, Burke RV, Lim J, et al. Pediatric traumaassociated acute respiratory distress syndrome: Incidence, risk factors, and outcomes. J Pediatr Surg 2019;54:1405-10.

37. Josephson CD, Mondoro TH, Ambruso DR, et al. One size will never fit all: the future of research in pediatric transfusion medicine. Pediatr Res 2014;76:425-31.

38. Kleiber N, Lefebvre É, Gauvin F, et al. Respiratory Dysfunction Associated With RBC Transfusion in Critically Ill Children: A Prospective Cohort Study. Pediatr Crit Care Med 2015;16:325-34.

39. Marik PE, Corwin HL. Acute lung injury following blood transfusion: expanding the definition. Crit Care Med 2008;36:3080-4.

40. De Cloedt L, Emeriaud G, Lefebvre É, et al. Transfusionassociated circulatory overload in a pediatric intensive care unit: different incidences with different diagnostic criteria. Transfusion 2018;58:1037-44.

41. De Cloedt L, Savy N, Gauvin F, et al. TransfusionAssociated Circulatory Overload in ICUs: A Scoping Review of Incidence, Risk Factors, and Outcomes. Crit
Care Med 2019;47:849-56.

42. Muszynski JA, Spinella PC, Cholette JM, et al. Transfusion-related immunomodulation: review of the literature and implications for pediatric critical illness. Transfusion 2017;57:195-206.

43. Parker RI. Transfusion in critically ill children: indications, risks, and challenges. Crit Care Med 2014;42:675-90.

44. Reynolds JD, Hess DT, Stamler JS. The transfusion problem: role of aberrant S-nitrosylation. Transfusion 2011;51:852-8.

45. Riccio DA, Zhu H, Foster MW, et al. Renitrosylation of banked human red blood cells improves deformability and reduces adhesivity. Transfusion 2015;55:2452-63.

46. Said AS, Doctor A. Influence of red blood cell-derived microparticles upon vasoregulation. Blood Transfus 2017;15:522-34.

47. Rogers SC, Moynihan FT, McDonough R, et al. Effect of plasma processing and storage on microparticle abundance, nitric oxide scavenging, and vasoactivity. Transfusion 2019;59:1568-77.

48. Lackritz EM, Campbell CC, Ruebush TK, et al. Effect of blood transfusion on survival among children in a Kenyan hospital. Lancet 1992;340:524-8.

49. Lackritz EM, Hightower AW, Zucker JR, et al. Longitudinal evaluation of severely anemic children in Kenya: the effect of transfusion on mortality and hematologic recovery. AIDS 1997;11:1487-94.

50. English M, Ahmed M, Ngando C, et al. Blood transfusion for severe anaemia in children in a Kenyan hospital. Lancet 2002;359:494-5.

51. Rajasekaran S, Sanfilippo D, Shoemaker A, et al. Respiratory Impairment after Early Red Cell Transfusion in Pediatric Patients with ALI/ARDS. Crit Care Res Pract 2012;2012:646473.

52. Church GD, Matthay MA, Liu K, et al. Blood product transfusions and clinical outcomes in pediatric patients with acute lung injury. Pediatr Crit Care Med 2009;10:297-302.

53. Proceedings of Réanimation 2018, the French Intensive Care Society International Congress. Ann Intensive Care 2018;8:13.

54. Demaret P, Emeriaud G, Hassan NE, et al. Recommendations on RBC Transfusions in Critically Ill Children With Acute Respiratory Failure From the Pediatric Critical Care Transfusion and Anemia Expertise Initiative. Pediatr Crit Care Med 2018;19:S114-20.

55. Valentine SL, Bembea MM, Muszynski JA, et al. Consensus Recommendations for RBC Transfusion 
Practice in Critically Ill Children From the Pediatric

Critical Care Transfusion and Anemia Expertise Initiative. Pediatr Crit Care Med 2018;19:884-98.

56. Markham C, Small S, Hovmand P, et al. Transfusion Decision Making in Pediatric Critical Illness. Pediatr Clin
North Am 2017;64:991-1015.

57. Díaz F, Nuñez MJ, Pino P, et al. Implementation of preemptive fluid strategy as a bundle to prevent fluid overload in children with acute respiratory distress syndrome and sepsis. BMC Pediatr 2018;18:207.

Cite this article as: François T, Emeriaud G, Karam O, Tucci M. Transfusion in children with acute respiratory distress syndrome. Ann Transl Med 2019;7(19):511. doi: 10.21037/ atm.2019.08.28 